\title{
Spin thermoelectrics in a disordered Fermi gas
}

\author{
J. Borge, ${ }^{1}$ C. Gorini, ${ }^{2}$ and R. Raimondi ${ }^{1}$ \\ ${ }^{1}$ CNISM and Dipartimento di Fisica "E. Amaldi", via della Vasca Navale 84, Università Roma Tre, 00146 Roma, Italy \\ ${ }^{2}$ Institut für Physik, Universität Augsburg, 86135 Augsburg, Germany
}

(Received 16 November 2012; published 14 February 2013)

\begin{abstract}
We study the connection between the spin-heat and spin-charge response in a disordered Fermi gas with spin-orbit coupling. It is shown that the ratio between the above responses can be expressed as the thermopower $S=-\left(\pi k_{B}\right)^{2} T \sigma^{\prime} / 3 e \sigma$ times a number $R_{s}$ which depends on the strength and type of the spin-orbit couplings considered. The general results are illustrated by examining different two-dimensional electron or hole systems with different and competing spin-orbit mechanisms, and we conclude that a metallic system could prove much more efficient as a heat-to-spin than as a heat-to-charge converter.
\end{abstract}

DOI: 10.1103/PhysRevB.87.085309

PACS number(s): 72.25.Ba, 72.20.Pa, 72.25.Dc, 72.25.Rb

\section{INTRODUCTION}

The moving carriers in a metallic system, electrons or holes, transport both electric charge and heat. This gives rise to a number of thermoelectric effects as well as a deep connection between thermal and electrical conductivities. A well known example is the Wiedemann-Franz law, which states that the ratio of the thermal to the electrical conductivity is the temperature times a universal number, the Lorenz number $\mathcal{L}=\pi^{2} k_{B}^{2} / 3 e^{2}$, where $k_{B}$ and $e$ are the Boltzmann constant and the unit charge, respectively. From the theoretical standpoint the validity of the above law relies on the singleparticle description of transport, on the Fermi statistics of carriers, and on the assumption of purely elastic scattering. ${ }^{1,2}$ When electron-electron interaction is present as in a Fermi liquid, this law still holds provided the quasiparticles do not exchange energy during collisions. At low temperatures the combination of electron-electron interaction and disorder may change this picture. $^{3-9}$ Additionally, a magnetic field affects both thermal and electrical transport yielding both galvanomagnetic and thermomagnetic effects. ${ }^{10}$ The above situation gets even more complicated when a third quantity transported by the carriers-the spin-is connected to the previous two by spin-orbit (SO) coupling. On the bright side, such a connection also opens up a plethora of new possibilities related to the manipulation of the additional spin degrees of freedom. This is testified by the recent rapid development of spintronics ${ }^{11,12}$ and spin caloritronics. ${ }^{13}$ A fundamental goal of spintronics is the achievement of all-electrical control of the carriers' spin, made possible by SO coupling as exemplified by the spin Hall effect. ${ }^{14-18}$ Similarly, an important goal of spin caloritronics is the manipulation of the spin degrees of freedom via thermal gradients, ${ }^{19-23}$ particularly relevant when energy efficiency issues are considered. ${ }^{13}$ In this context a noteworthy phenomenon is the spin Seebeck effect: a spin current thermally generated in a (metallic or insulating) ferromagnet is injected into a normal metal, and there, via the inverse spin Hall effect, it generates an observable voltage drop in the direction orthogonal to the applied thermal gradient. ${ }^{24-26}$ In this much studied case phonons and magnons play the leading roles. ${ }^{27-29}$ There are on the other hand only few works on thermo-spin transport due to the charge carriers' dynamics, ${ }^{20,21}$ and we wish to address this issue considering disordered Fermi gases with SO coupling. We will see that a general relation between the spin-heat and spin-charge response of such systems can be obtained, with the same range of applicability of the Wiedemann-Franz law. Moreover, we will discuss the particular case of the thermo-spin Hall effectthe generation of a spin current transverse to a thermal gradient, also called the spin Nernst effect. In so doing we will show that a simple relation connects the spin thermopower- the ratio between the spin response to a thermal gradient and that to an electric field - to the standard electric thermopower, and that the former can be strongly enhanced by the interplay between different SO coupling mechanisms.

Let us start with some basic phenomenological considerations along the lines of Refs. 30 and 31, and consider the bare-bones situation of an inversion symmetric, homogeneous, and nonferromagnetic material in the absence of magnetic fields. A particle current $j_{x}$ can be driven either by an electric field or by a temperature gradient, and within the standard semiclassical approach one writes ${ }^{32}$

$$
j_{x}=L_{11} E_{x}+L_{12}\left(-\nabla_{x} T\right)=\sigma E_{x}-e \mathcal{L} T \sigma^{\prime}\left(-\nabla_{x} T\right) .
$$

Here $\sigma=-2 e N_{0} D$ is the Drude conductivity up to a charge $-e$, with $N_{0}$ the density of states at the Fermi energy and $D$ the diffusion constant, and $\sigma^{\prime}=\partial_{\mu} \sigma, \mu$ being the chemical potential. The ratio $S \equiv L_{12} / L_{11}$ is the electric thermopower. In the present simple case the connection between spin and particle currents due to $\mathrm{SO}$ coupling reads ${ }^{31}$

$$
j_{y}^{z}=-\gamma j_{x}=L_{11}^{s} E_{x}+L_{12}^{s}\left(-\nabla_{x} T\right) .
$$

Here $j_{y}^{z}$ is the $z$-polarized spin current flowing in the $y$ direction arising in response to the particle current $j_{x}$, and $\gamma \ll 1$ is a dimensionless SO coupling constant. As an immediate consequence of Eqs. (1) and (2), the spin thermopower $S_{s} \equiv L_{12}^{s} / L_{11}^{s}$ is equal to $S$, since the SO coupling constant $\gamma$ does not depend on the sources of a given particle current. Equation (2) breaks down in the absence of inversion symmetry, and in order to see how the above simple result is modified in a general situation, and to study its dependence on competing SO coupling mechanisms, we will move on to a microscopic treatment.

The paper is organized as follows. The formalism is introduced in Sec. II and put to use in Sec. III to obtain the general formula for the spin-thermopower $S_{s}$. The latter appears as the spin equivalent of Mott's formula for the 
electric thermopower. In order to lend concreteness to the presentation, the derivation of $S_{s}$ is done using the linear Rashba model as a template. In Sec. IV we apply our formula to a series of different systems and discuss its experimental relevance, before concluding in Sec. V. A number of technical details regarding the Matsubara technique are presented in the Appendix.

\section{THE BASIC EQUATIONS}

Though our treatment is independent of dimensions (two or three dimensions), in order to fix things we consider a disordered two-dimensional (2D) Fermi gas in the $x-y$ plane described by the Hamiltonian

$$
H=\frac{p^{2}}{2 m}+V(\mathbf{x})+H_{\text {so }},
$$

with $\mathbf{p}$ the $2 \mathrm{D}$ momentum and $V(\mathbf{x})$ the impurity potential. For the latter we assume the standard white noise disorder model and evaluate the impurity average in the Born approximation, $\left\langle V(\mathbf{x}) V\left(\mathbf{x}^{\prime}\right)\right\rangle=\left(2 \pi N_{0} \tau\right)^{-1} \delta\left(\mathbf{x}-\mathbf{x}^{\prime}\right)$, with $N_{0}=$ $m /\left(2 \pi \hbar^{2}\right)$ and $\tau$ the elastic scattering time. The SO term $H_{\text {so }}$ will have different forms in the various cases considered below. In the (linear) Rashba case it reads

$$
H_{\text {so }}=\alpha \boldsymbol{\sigma} \cdot \mathbf{p} \times \hat{\mathbf{e}}_{z},
$$

with $\alpha$ a coupling constant. We assume the metallic regime and weak SO coupling conditions, $\epsilon_{F} \gg \hbar / \tau, \Delta_{\text {so }}$. Here $\epsilon_{F}$ is the Fermi energy in the absence of disorder and SO interaction, and $\Delta_{\mathrm{so}}$ is the SO splitting due to $H_{\mathrm{so}}$. From now on $\hbar, k_{B}=1$. The $a$-polarized spin current flowing in the $k$-direction due to a generic thermal gradient is

$$
j_{k}^{a}=\sum_{l}\left[\mathrm{~N}_{\mathrm{sh}}\right]_{k l}^{a}\left(-\partial_{l} T\right),
$$

where $\mathrm{N}_{\text {sh }}$ is the spin-heat response tensor. Following Ref. 7 the latter is given in terms of the imaginary spin current-heat current kernel

$$
\left[\mathrm{N}_{\mathrm{sh}}\right]_{k l}^{a} T=\lim _{\Omega \rightarrow 0}\left\{\frac{\left[Q_{\mathrm{sh}}\left(\mathrm{i} \Omega_{v}\right)\right]_{k l}^{a}}{\Omega_{v}}\right\}_{\mathrm{i} \Omega_{v} \rightarrow \Omega^{R}, \Omega^{R}=\Omega+\mathrm{i} 0^{+}} .
$$

The spin current operator is given by the standard definition $j_{k}^{a}=(1 / 2)\left\{v_{k}, s^{a}\right\}, v_{k}$ and $s^{a}$ being the velocity and spin operators, respectively. Notice that the particle (charge) current operator is $(-e) j_{k}=(-e) v_{k}$. The heat current in the Matsubara representation reads

$$
j_{k}^{h}\left(\mathbf{p}, \epsilon_{n}, \epsilon_{n}+\Omega_{v}\right)=\mathrm{i} \epsilon_{n+v / 2} j_{k},
$$

with $\epsilon_{n}=\pi T(2 n+1), \Omega_{v}=2 \pi T \nu$, and $\epsilon_{n+v / 2}=\epsilon_{n}+$ $\Omega_{v} / 2$. The specific form of $v_{k}$ depends on the choice of the SO Hamiltonian. For instance in the Rashba case, Eq. (4), we have $v_{x, y}=p_{x, y} / m \mp \alpha \sigma^{y, x}$. By using the Kubo formula the response kernel is given by

$$
\left[Q_{\mathrm{sh}}\right]_{k l}^{a}\left(\mathrm{i} \Omega_{\nu}\right)=T \sum_{\epsilon_{n}, \mathbf{p}} \mathrm{i} \epsilon_{n+v / 2} \operatorname{Tr}\left[j_{k}^{a} \mathcal{G}_{n} j_{l} \mathcal{G}_{n+v}\right],
$$

where the Matsubara Green functions $\mathcal{G}_{n}=\mathcal{G}\left(\mathbf{p}, \epsilon_{n}\right)$ and $\mathcal{G}_{n+v}=\mathcal{G}\left(\mathbf{p}, \epsilon_{n}+\Omega_{v}\right)$ are matrices in spin space $\mathcal{G}_{n}=\mathcal{G}_{n}^{0}+$ $\sum_{a} \mathcal{G}_{n}^{a} \sigma^{a}$. Analogously, the spin-charge response kernel can be written as

$$
\left[Q_{\mathrm{sc}}\right]_{k l}^{a}\left(\mathrm{i} \Omega_{\nu}\right)=-e T \sum_{\epsilon_{n}, \mathbf{p}} \operatorname{Tr}\left[j_{k}^{a} \mathcal{G}_{n} j_{l} \mathcal{G}_{n+v}\right],
$$

leading to the spin-charge (particle) conductivity

$$
\left[\sigma_{\mathrm{sc}}\right]_{k l}^{a}=\lim _{\Omega \rightarrow 0}\left\{\frac{\left[Q_{\mathrm{sc}}\left(\mathrm{i} \Omega_{v}\right)\right]_{k l}^{a}}{\Omega_{v}}\right\}_{\mathrm{i} \Omega_{v} \rightarrow \Omega^{R}, \Omega^{R}=\Omega+\mathrm{i} 0^{+}} .
$$

\section{THE SPIN EQUIVALENT OF MOTT'S FORMULA}

Although our treatment is general, to illustrate the procedure, we take the Rashba case as an example. The average over disorder is evaluated in the Born approximation and leads to a self-energy

$$
\Sigma\left(\epsilon_{n}\right)=\frac{1}{2 \pi N_{0} \tau} \sum_{\mathbf{p}} \mathcal{G}_{n}=-\frac{\mathrm{i}}{2 \tau} \operatorname{sgn}\left(\epsilon_{n}\right),
$$

which is diagonal in spin space. As it can be seen from Eq. (4) for the Rashba case, the off-diagonal terms in spin space of the Green function are odd in the momentum dependence and vanish upon integration. This remains valid also for other spin-orbit interaction terms as long as the Hamiltonian is timereversal invariant.

To compute the thermo-spin Hall effect, i.e., the $z$-polarized spin current flowing along $y$ generated by a thermal gradient along $x$, we need the response kernel $\left[Q_{\mathrm{sh}}\right]_{y x}^{z} \equiv Q^{s H}$, which reads

$$
Q^{s H}\left(\mathrm{i} \Omega_{v}\right)=T \sum_{\epsilon_{n}} \sum_{\mathbf{p}} \mathrm{i} \epsilon_{n+v / 2} \operatorname{Tr}\left[j_{y}^{z} \mathcal{G}_{n} j_{x} \mathcal{G}_{n+v}\right],
$$

with $\mathcal{G}_{n}^{0}=\left(\mathcal{G}_{n,+}+\mathcal{G}_{n,-}\right) / 2 \quad$ and $\quad \mathcal{G}_{n}^{a}=\left(\hat{\mathbf{p}} \times \hat{\mathbf{e}}_{z}\right)^{a}\left(\mathcal{G}_{n,+}-\right.$ $\left.\mathcal{G}_{n,-}\right) / 2$, whereas

$$
\mathcal{G}_{n, \pm}=\left[\mathrm{i} \epsilon_{n}+\mu-\frac{p^{2}}{2 m} \mp \alpha p+\frac{\mathrm{i}}{2 \tau} \operatorname{sgn}\left(\epsilon_{n}\right)\right]^{-1},
$$

$\mu$ being the chemical potential.

Notice that the analytic properties of the Green functions are determined by the sign of the imaginary frequency; therefore when performing the momentum integral in Eq. (12) one obtains a nonzero result only if the frequencies $\epsilon_{n}+\Omega_{v}$ and $\epsilon_{n}$ have opposite signs, which means that $\epsilon_{n}$ is restricted to the range $-\Omega_{v}<\epsilon_{n}<0$. Exploiting that the external frequency is going to zero [cf. Eq. (6)], one thus has

$$
\sum_{\mathbf{p}} \operatorname{Tr}\left[j_{k}^{a} \mathcal{G}_{n} j_{l} \mathcal{G}_{n+\nu}\right]=-\frac{2 \pi}{e}\left[\sigma_{\mathrm{sc}}\right]_{k l}^{a}\left(\mu+\mathrm{i} \epsilon_{n}\right) .
$$

This last result is derived in the Appendix more in detail.

Equation (12) only takes into account the so-called bare bubble. Vertex corrections ${ }^{33,34}$ will be considered later. According to Eq. (14) we now have

$$
\sum_{\mathbf{p}} \operatorname{Tr}\left[j_{y}^{z} \mathcal{G}_{n} j_{x} \mathcal{G}_{n+v}\right]=-\frac{2 \pi}{e} \sigma^{s H}\left(\mu+\mathrm{i} \epsilon_{n}\right),
$$


with $\sigma^{s H}(\mu)$ the static spin-Hall conductivity from Ref. 35. The thermo-spin Hall conductivity, therefore, reads

$$
\mathrm{N}^{s H} T=-\lim _{\Omega \rightarrow 0}\left[\frac{2 \pi T}{e \Omega_{v}} \sum_{n=-v}^{-1} \mathrm{i} \epsilon_{n+v / 2} \sigma^{s H}\left(\mu+\mathrm{i} \epsilon_{n}\right)\right]_{\mathrm{i} \Omega_{v} \rightarrow \Omega^{R}},
$$

and, after expanding in $\epsilon_{n}$ as shown in the Appendix, yields

$$
\left.\mathrm{N}^{s H}\right|_{\text {bare }}=-\frac{\pi^{2} T}{3} \frac{m \alpha^{2} \tau^{2}}{\pi} .
$$

To connect this result with that of Ref. 20, in which $\mathrm{N}^{s H} T$ is computed in the clean limit, $\tau \rightarrow \infty$, we rewrite Eq. (13) as

$$
\mathcal{G}_{n, \pm}=\left[\mu-\frac{p^{2}}{2 m} \mp \alpha p+\mathrm{i} \operatorname{sgn}\left(\epsilon_{n}\right)\left(\frac{1}{2 \tau}+\left|\epsilon_{n}\right|\right)\right]^{-1},
$$

and note that, as long as the temperature is finite, in the $\tau \rightarrow \infty$ limit the poles are $2 \pi T$ away from the real axis. Thus the effective replacement $1 / 2 \tau \rightarrow \pi T$ in Eq. (17) yields the clean limit result

$$
\left.\mathrm{N}^{s H}\right|_{\text {clean }}=-\frac{m \alpha^{2}}{12 \pi T},
$$

in agreement with Ref. 20. Let us now discuss the vertex corrections. Taking them into account corresponds to sending $j_{y}^{z} \rightarrow J_{y}^{z}, j_{x} \rightarrow J_{x}$, and $j_{x}^{h} \rightarrow J_{x}^{h}$. At the level of the Born approximation either vertex could be renormalized: the bubble with $J_{y}^{z}$ and $j_{x}^{h}$ or that with $j_{y}^{z}$ and $J_{x}^{h}$ are equivalent. Moreover, since we neglect inelastic processes, $J_{x}^{h}=\mathrm{i} \epsilon_{n+v / 2} J_{x}$. For the Rashba case it is known that $J_{x}=0$, i.e., $\sigma^{s H}=0$, and thus we immediately obtain

$$
\left.\mathrm{N}^{s H}\right|_{\text {dressed }}=0 .
$$

However, notice that Eq. (16) holds for any form of the SO interaction term $H_{\text {so }}$, no matter whether of intrinsic or extrinsic nature. Therefore, once the spin-Hall conductivity $\sigma^{s H}$ of a given system is known, its thermo-spin Hall conductivity $\mathrm{N}^{s H}$ will follow at once. Even more generally, from the Matsubara formulation, Eqs. (6)-(14), we conclude that the spin-heat response of a disordered, SO coupled Fermi gas in the metallic regime is completely determined by its spincharge response. This result holds in two and three dimensions, in the presence of arbitrary elastic scattering processes, possibly spin-dependent, and beyond the Born approximation, i.e., it has the same range of applicability of the Wiedemann-Franz law discussed in Ref. 7. This is the first main result of our work, which, after a Sommerfeld expansion, can be written in the very simple form

$$
\mathrm{N}_{\mathrm{sh}}=-e \mathcal{L} T \sigma_{\mathrm{sc}}^{\prime}(\mu) .
$$

In other words Mott's formula for the electric thermopower $S=-e \mathcal{L} T \sigma^{\prime} / \sigma$ has its symmetric spin equivalent

$$
S_{s}=-e \mathcal{L} T \sigma_{\mathrm{sc}}^{\prime} / \sigma_{\mathrm{sc}} .
$$

Whether a direct relation between $S_{s}$ and $S$ exists is, however, not obvious and will be one of our next concerns.

\section{SPIN NERNST EFFECT AND SPIN THERMOPOWER IN ELECTRON AND HOLE GASES}

Specializing our treatment to some specific systems, we now have a two-fold aim: (i) to look for the possibility of efficient heat-to-spin conversion, $S_{S} \gg 1$; (ii) to establish a relation, if any, between $S_{s}$ and $S$.

With this in mind, let us now take $H_{\mathrm{so}}$ to be linear in momentum, in which case the spin continuity equations assume a particularly simple form. This allows one to easily draw a set of more specific conclusions concerning the thermospin response of the 2D Fermi gas, in particular regarding the interplay between different $\mathrm{SO}$ and scattering mechanisms. To be explicit we take once more the disordered Rashba model as the initial example, and consider the presence of extrinsic SO mechanisms and (white noise) magnetic impurities. That is, we add to the Hamiltonian (3) the terms

$$
H_{\mathrm{extr}}=-\frac{\lambda_{0}^{2}}{4} \boldsymbol{\sigma} \times \nabla V(\mathbf{x}) \cdot \mathbf{p},
$$

with $\lambda_{0}$ an effective Compton wavelength, and

$$
V_{\mathrm{m}}(\mathbf{x})=\sum_{\mathbf{i}} \mathbf{B} \cdot \boldsymbol{\sigma} \delta\left(\mathbf{x}-\mathbf{R}_{\mathbf{i}}\right)
$$

where $\mathbf{B}$ is a random (white noise) magnetic field. The latter is handled in the Born approximation, $\left\langle V_{\mathrm{m}}(\mathbf{x}) V_{\mathrm{m}}\left(\mathbf{x}^{\prime}\right)\right\rangle=$ $\left[3\left(2 \pi N_{0} \tau_{\mathrm{sf}}\right)\right]^{-1} \delta\left(\mathbf{x}-\mathbf{x}^{\prime}\right)$, with $\tau_{\mathrm{sf}}$ the spin-flip time. ${ }^{36,37}$ The $s^{y}$ continuity equation reads

$$
\partial_{t} s^{y}+\nabla \cdot \mathbf{j}^{y}=-2 m \alpha j_{y}^{z}-\left(\frac{4}{3 \tau_{\mathrm{sf}}}+\frac{1}{\tau_{\mathrm{EY}}}\right) s^{y},
$$

with $\tau_{\mathrm{EY}}=\tau\left(\lambda_{0} p_{F} / 2\right)^{-4}$ the Elliot-Yafet spin-relaxation time due to $H_{\text {extr }}$. Assuming a homogeneous electric field applied in the $x$ direction, the spin current $j_{y}^{z}$ in the diffusive regime is given by

$$
j_{y}^{z}=2 m \alpha D s_{y}-\gamma \sigma E_{x},
$$

where $\gamma=\gamma_{\text {intr }}+\gamma_{\mathrm{sj}}+\gamma_{\mathrm{ss}}$ is the SO coupling constant due to intrinsic and extrinsic mechanisms, with $\gamma_{\text {intr }}=$ $-m \alpha^{2} \tau, \gamma_{\mathrm{sj}}=\left(\lambda_{0} / 2\right)^{2} m / \tau$ the side-jump contribution, and $\gamma_{\mathrm{ss}}=\left(\lambda_{0} p_{F} / 4\right)^{2}\left(2 \pi N_{0} v_{0}\right)$ the skew-scattering one. $v_{0}$ is the scattering amplitude (see Refs. 38 and 39 for details). In a homogeneous bulk in steady state the spin-Hall conductivity is easily computed, $\sigma^{s H}=[1 /(1+\zeta)] \gamma \sigma$, where $\zeta \equiv \tau_{\mathrm{s}} / \tau_{\mathrm{DP}}$, with $1 / \tau_{\mathrm{DP}}=(2 m \alpha)^{2} D$ the Dyakonov-Perel spin-relaxation rate, and $1 / \tau_{\mathrm{s}} \equiv 4 /\left(3 \tau_{\mathrm{sf}}\right)+1 / \tau_{\mathrm{EY}}$. Via Eq. (21) one concludes

$$
\begin{aligned}
& \sigma^{\prime s H}=\left[\frac{\sigma^{\prime}}{\sigma}+\frac{\gamma^{\prime}}{\gamma}-\frac{\zeta^{\prime}}{1+\zeta}\right] \sigma^{s H}, \\
& S_{s}=-e \mathcal{L} T\left[\frac{\sigma^{\prime}}{\sigma}+\frac{\gamma^{\prime}}{\gamma}-\frac{\zeta^{\prime}}{1+\zeta}\right],
\end{aligned}
$$

with the spin Hall thermopower $S_{s}=\mathrm{N}^{s H} / \sigma^{s H}$. In the above, primed quantities are derivatives with respect to the chemical potential $\mu$. Notice that the simple phenomenological argument of the introduction overlooks the $\mu$ dependency of $\gamma$ : the conclusion $S_{s}=S$ holds only for an energy-independent $\gamma$. Both $\sigma^{s H}$ and $\mathrm{N}^{s H}$ depend on the ratio between $\tau_{\mathrm{DP}}$ and $\tau_{\mathrm{s}}$ and are in principle tunable, either by varying the doping, which affects $\tau_{\mathrm{s}}$, or by modulating $\alpha$ by varying the gate potential. Let us consider some interesting cases using Eqs. (27) and (28). 
When only Rashba SO and magnetic impurities are present, we have $\tau_{\mathrm{s}}=3 \tau_{\mathrm{sf}} / 4$ and $\gamma=\gamma_{\text {int }}$. By evaluating the various derivatives we obtain $\gamma^{\prime}=0, \zeta^{\prime}=\zeta / \mu$, and $\sigma^{\prime}=\sigma / \mu$, which gives us the spin thermopower

$$
S_{s}=-e \mathcal{L} T \frac{\sigma^{\prime}}{\sigma} \frac{1}{1+\zeta} .
$$

When SO from impurities is present, too, the terms $\gamma^{\prime} / \gamma$ and $\zeta^{\prime} / \zeta$ in Eq. (27) are modified, leading to

$$
S_{s}=-e \mathcal{L} T \frac{\sigma^{\prime}}{\sigma}\left[1+\frac{\gamma_{\mathrm{ss}}}{\gamma}-\frac{\zeta}{1+\zeta}\left(1-\frac{2 \tau_{\mathrm{s}}}{\tau_{\mathrm{EY}}}\right)\right] .
$$

The results so far obtained can be generalized to include the effects of the linear-in-momentum Dresselhaus SO term described by the Hamiltonian

$$
H_{\text {so }}=\beta\left(p_{x} \sigma^{x}-p_{y} \sigma^{y}\right) .
$$

It suffices to replace in the above $\gamma_{\text {intr }}=-m \tau\left(\alpha^{2}-\right.$ $\left.\beta^{2}\right), 1 / \tau_{\mathrm{DP}}=(2 m)^{2}\left(\alpha^{2}+\beta^{2}\right) D \equiv 1 / \tau_{\mathrm{DP}}^{R}+1 / \tau_{\mathrm{DP}}^{D}$, and

$$
\zeta=\frac{\tau_{\mathrm{s}}}{\tau_{\mathrm{DP}}}-4 \frac{\tau_{\mathrm{s}}^{2} /\left(\tau_{\mathrm{DP}}^{R} \tau_{\mathrm{DP}}^{D}\right)}{\tau_{\mathrm{s}} / \tau_{\mathrm{DP}}+1} .
$$

The derivatives are trivial, but yield expressions too cumbersome to be conveniently written down. The results are thus plotted in Fig. 1, and show the sensitivity of the spin
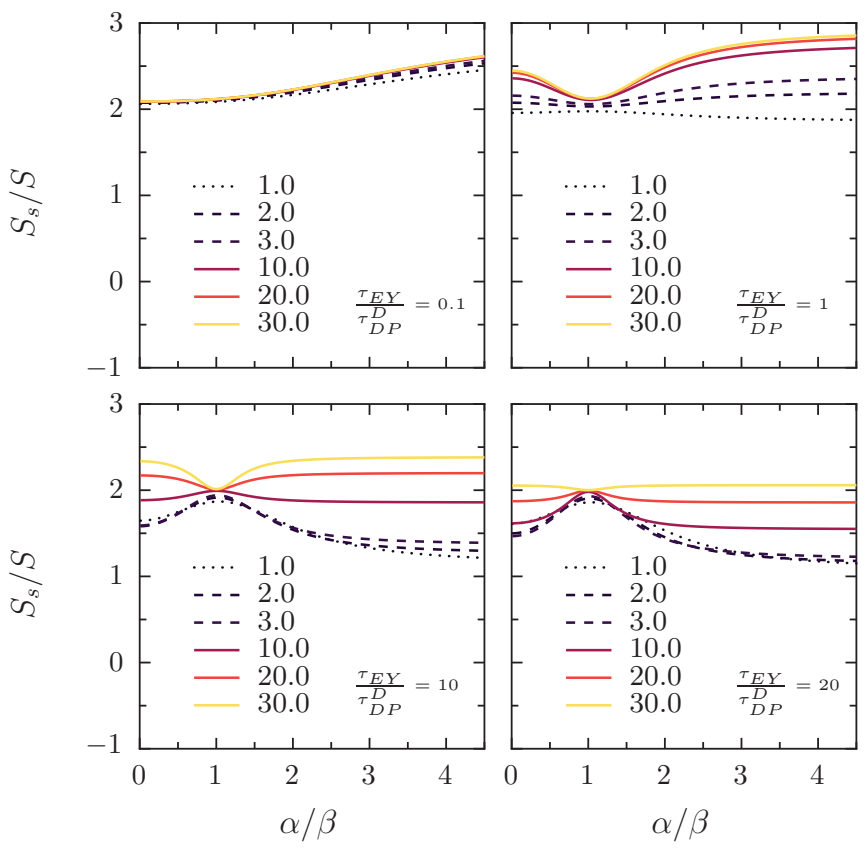

FIG. 1. (Color online) The spin thermopower $S_{s}$ of a disordered 2D-electron gas with numerous competing SO mechanisms. Typical values for GaAs quantum wells are mobility $\mu=10^{4} \mathrm{~cm}^{2} / \mathrm{Vs}$, density $n=10^{12} \mathrm{~cm}^{-2}$, effective extrinsic wavelength $\lambda_{0}=4.7 \times 10^{-8} \mathrm{~cm}$, and Dresselhaus coupling constant $\hbar \beta=10^{-12} \mathrm{eVm}$. There follows $\gamma_{\mathrm{ss}} \gg \gamma_{\text {intr }}, \gamma_{s j}, \tau_{\mathrm{EY}} \gg \tau_{\mathrm{DP}}^{D}$. The Rashba coupling constant can be modulated by the gate potential. ${ }^{40,41}$ Each panel shows the ratio $S_{s} / S$ as a function of the ratio $\alpha / \beta$ for a given Elliot-Yafet scattering strength, strong to weak from top left to bottom right-panel 3 corresponds to standard GaAs. Magnetic scattering is strongest for the dotted curve, $\tau_{s f} / \tau_{\mathrm{DP}}^{D}=1$, and strong (weak) for the dashed (solid) curves, $\tau_{s f} / \tau_{\mathrm{DP}}^{D}=2,3(10,20,30)$. thermopower to the various physical parameters in play. A modest modulation of the Rashba coupling constant could substantially modify $S_{s}$, either enhancing or decreasing it depending on the systems characteristics - we considered ratios $\alpha / \beta$ well within current experimental capabilities. ${ }^{40,41}$ We will come back to this point in a moment. Let us now consider our final example, a 2D hole gas as analyzed in Ref. 42. The SO interaction is cubic in momentum

$$
H_{\mathrm{so}}=\alpha_{H} \sigma_{x}\left[p_{y}\left(3 p_{x}^{2}-p_{y}^{2}\right)\right]+\alpha_{H} \sigma_{y}\left[p_{x}\left(3 p_{y}^{2}-p_{x}^{2}\right)\right],
$$

and the spin Hall conductivity reads ${ }^{42}$

$$
\sigma_{H}^{s H}=-\frac{3 \eta^{2}\left(4 \eta^{2}-1\right)}{\left(4 \eta^{2}+1\right)^{2}} \frac{1}{\mu \tau} \sigma,
$$

with $\eta=\alpha_{H} p_{F}^{3} \tau$ (Ref. 43). Proceeding as before one gets

$$
S_{s}=-e \mathcal{L} T \frac{\sigma^{\prime}}{\sigma}\left[\frac{3\left(12 \eta^{2}-1\right)}{\left(4 \eta^{2}+1\right)\left(4 \eta^{2}-1\right)}\right] .
$$

All previous result can be cast in the simple form

$$
S_{S}=S R_{s},
$$

with $R_{S}$ a number which depends on the various competing SO mechanisms. Equation (36), which is our second main result, looks physically quite reasonable: in a metallic system in which electrons (or holes) are the sole carriers of charge, spin and heat, the heat-to-spin and heat-to-charge (particle) conversions are expected to be closely related. The examples considered show however that $R_{s}>1$ could be easily achieved: in standard GaAs samples with Rashba and extrinsic SO mechanisms, one may estimate $R_{s} \sim 3$ (Ref. 38), and the same value is obtained in a two-dimensional hole gas with purely cubic Rashba SO in the diffusive regime $(\eta \ll 1)$. If Dresselhaus SO is also taken into account, similar values could be achieved, as shown in Fig. 1. This suggests that metallic systems, typically characterized by low thermoelectric efficiencies, could be much more efficient in heat-to-spin conversion and therefore play a front role in spin caloritronics. Of course, whether substantially higher $R_{s}$ values can be reached in different systems, e.g., in transition metals which already show a giant spin Hall response, ${ }^{44,45}$ or more exotic ones such as $p$-doped graphene, ${ }^{46}$ or topological insulators like $\mathrm{HgTe},{ }^{47}$ is an open and relevant question. Indeed, it would be interesting to establish whether it is always possible, within the regime in which the general expression (21) holds, to find such a simple connection between $S_{s}$ and $S$. We, therefore, believe it desirable to experimentally test Eq. (36). This could be done rather straightforwardly in a setup like the one employed to first observe the spin Hall effect: ${ }^{48}$ at low temperatures, the spin accumulation at the side edges of a two-dimensional Fermi gas could be optically measured first in response to a longitudinally applied bias, and then to a small temperature gradient along the same direction. All-electrical measurement schemes based on H-bar geometries, exchanging again the applied bias with a temperature difference, would also be interesting though probably more delicate: in this case a temperature gradient along the side leg of the H-bar should be avoided or its effects compensated. Finally, it is well known that Mott's formula 
can be heavily affected by inelastic processes. Though the latter are beyond the scope of the present work, it would be interesting to study their effects on $S_{s}$ and see whether any similarities between electric and spin thermopower exist also in their presence or not.

\section{CONCLUSIONS}

In conclusion, we have studied coupled spin and thermal transport in a disordered and SO coupled Fermi gas, and shown the existence of a general expression for the spin thermopower $S_{S}$ with the same structure and an identical range of validity of Mott's formula for the electric thermopower $S$. Finally, we have derived a simple and physically transparent relation connecting the two quantities which could be experimentally tested and suggests that metallic systems could be much more efficient in heat-to-spin than in heat-to-charge conversion.

\section{ACKNOWLEDGMENTS}

We acknowledge financial support from the EU through Grant. No. PITN-GA-2009-234970 and from the German Research Foundation DFG (TRR80). C.G. thanks G.-L. Ingold for PYX support.

\section{APPENDIX: DERIVATION OF EQS. (14) AND (17)}

By defining

$$
F\left(\mathrm{i} \epsilon_{n}, \mathrm{i} \Omega_{v}\right)=\sum_{\mathbf{p}} \operatorname{Tr}\left[j_{k}^{a} \mathcal{G}_{n} j_{l} \mathcal{G}_{n+v}\right],
$$

we write the spin-heat and spin-charge responses as

$$
\begin{aligned}
\sigma_{\mathrm{sc}} & =\lim _{\Omega \rightarrow 0}\left\{\frac{(-e) T}{\Omega_{v}} \sum_{\epsilon_{n}} F\left(\mathrm{i} \epsilon_{n}, \mathrm{i} \Omega_{v}\right)\right\}_{\mathrm{i} \Omega_{v} \rightarrow \Omega^{R}}, \\
N_{\mathrm{sh}} & =\lim _{\Omega \rightarrow 0}\left\{\frac{1}{\Omega_{v}} \sum_{\epsilon_{n}} \mathrm{i} \epsilon_{n+v / 2} F\left(\mathrm{i} \epsilon_{n}, \mathrm{i} \Omega_{v}\right)\right\}_{\mathrm{i} \Omega_{v} \rightarrow \Omega^{R}} .
\end{aligned}
$$

As mentioned in the main text, the momentum integral yields a nonzero result only if the frequencies $\epsilon_{n}+\Omega_{v}$ and $\epsilon_{n}$ have opposite signs, which means that $\epsilon_{n}$ is restricted to the range $-\Omega_{v}<\epsilon_{n}<0$. Since the external frequency is going to zero, so will $\mathrm{i} \epsilon_{n}$, enabling one to expand $F$ in powers of $\mathrm{i} \epsilon_{n}$ as

$$
F\left(\mathrm{i} \epsilon_{n}, \mathrm{i} \Omega_{v}\right)=F\left(0, \mathrm{i} \Omega_{v}\right)+\mathrm{i} \epsilon_{n} \frac{\partial F}{\partial \mathrm{i} \epsilon_{n}}\left(0, \mathrm{i} \Omega_{v}\right)+\cdots .
$$

Replacing this expansion in Eq. (A2) we have

$$
\begin{aligned}
\sigma_{\mathrm{sc}}= & \lim _{\Omega \rightarrow 0}\left\{\frac{-e T}{\Omega_{v}} \sum_{n=-v}^{-1} F\left(0, \mathrm{i} \Omega_{v}\right)\right. \\
& \left.+\mathrm{i} \epsilon_{n} \frac{\partial F}{\partial \mathrm{i} \epsilon_{n}}\left(0, \mathrm{i} \Omega_{v}\right)+\cdots\right\}_{\mathrm{i} \Omega_{v} \rightarrow \Omega^{R}} .
\end{aligned}
$$

The first term of the sum is linear in $\Omega_{v}$, so when divided by $\Omega_{v}$ in the zero-frequency limit it yields a nonzero contribution. The other terms of the sum, being at least quadratic in $\Omega_{v}$, clearly do not contribute. There follows

$$
\sigma_{\mathrm{sc}}=-\frac{e}{2 \pi} F(0,0) \text {. }
$$

This is enough to prove Eq. (14). To prove Eq. (17), we expand Eq. (A3) in $\mathrm{i} \epsilon_{n}$ and note that the zero order term of the sum vanishes since

$$
\sum_{-\Omega_{\nu}<\epsilon_{n}<0}\left(\mathrm{i} \epsilon_{n}+\frac{\mathrm{i} \Omega_{v}}{2}\right)=0 .
$$

By noticing that

$$
\sum_{-\Omega_{v}<\epsilon_{n}<0}\left(\mathrm{i} \epsilon_{n}+\frac{\mathrm{i} \Omega_{v}}{2}\right) \mathrm{i} \epsilon_{n}=\frac{\pi^{2} T^{2}}{3} v\left(1-v^{2}\right),
$$

the only term contributing linearly in $\Omega_{v}$ is the first order one. This leads to

$$
\mathrm{N}_{\mathrm{sh}}=-e \mathcal{L} T F^{\prime}(0,0),
$$

with $\mathcal{L}$ the Lorenz number and $F^{\prime}=\frac{\partial F}{\partial \epsilon_{n}}$. The last step in proving Eq. (17) of the main text is the observation that the function $F$ of Eq. (A1) depends on $\epsilon_{n}$ through the combination $\mathrm{i} \epsilon_{n}+\mu$, as it is evident from the expression of the Green functions in the restricted frequency range $-\Omega_{v}<\epsilon_{n}<0$

$$
\begin{gathered}
\mathcal{G}_{n}=\left[\mathrm{i} \epsilon_{n}+\mu-\frac{\mathrm{i}}{2 \tau}-H_{\mathrm{so}}\right]^{-1}, \\
\mathcal{G}_{n+v}=\left[\mathrm{i}\left(\epsilon_{n}+\Omega_{v}\right)+\mu+\frac{\mathrm{i}}{2 \tau}-H_{\mathrm{so}}\right]^{-1},
\end{gathered}
$$

where we have left unspecified the spin-orbit Hamiltonian for the sake of generality.
${ }^{1}$ G. V. Chester and A. Thellung, Proc. Phys. Soc. London 77, 1005 (1961).

${ }^{2}$ M. Jonson and G. D. Mahan, Phys. Rev. B 21, 4223 (1980).

${ }^{3}$ J. S. Langer, Phys. Rev. 128, 110 (1962).

${ }^{4}$ C. Castellani, C. Di Castro, G. Kotliar, P. A. Lee, and G. Strinati, Phys. Rev. Lett. 59, 477 (1987).

${ }^{5}$ D. V. Livanov, M. Y. Reizer, and A. V. Sergeev, Zh. Eksp. Teor. Fiz. 99, 1230 (1991) [Sov. Phys. JETP 72, 760 (1991)].

${ }^{6}$ R. Raimondi, G. Savona, P. Schwab, and T. Lück, Phys. Rev. B 70, 155109 (2004).
${ }^{7}$ D. R. Niven and R. A. Smith, Phys. Rev. B 71, 035106 (2005).

${ }^{8}$ G. Catelani and I. L. Aleiner, Zh. Eksp. Teor. Fiz. 127, 372 (2005) [Sov. Phys. JETP 100, 331 (2005)].

${ }^{9}$ K. Michaeli and A. M. Finkelstein, Phys. Rev. B 80, 115111 (2009).

${ }^{10}$ E. H. Sondheimer, Proc. R. Soc. London A 193, 484 (1948).

${ }^{11}$ I. Žutić, J. Fabian, and S. D. Sarma, Rev. Mod. Phys. 76, 323 (2004).

${ }^{12}$ D. D. Awschalom and M. E. Flatté, Nat. Phys. 3, 153 (2007).

${ }^{13}$ G. E. W. Bauer, E. Saitoh, and B. J. van Wees, Nat. Mater. 11, 391 (2012).

${ }^{14}$ M. I. Dyakonov and V. I. Perel, Phys. Lett. A 35, 459 (1971). 
${ }^{15}$ J. E. Hirsch, Phys. Rev. Lett. 83, 1834 (1999).

${ }^{16}$ S. Murakami, N. Nagaosa, and S.-C. Zhang, Science 301, 1348 (2003).

${ }^{17}$ J. Sinova, D. Culcer, Q. Niu, N. A. Sinitsyn, T. Jungwirth, and A. H. MacDonald, Phys. Rev. Lett. 92, 126603 (2004).

${ }^{18}$ G. Vignale, J. Supercond. Nov. Magn. 23, 3 (2010).

${ }^{19}$ C. M. Wang and M. Q. Pang, Solid State Commun. 150, 1509 (2010).

${ }^{20}$ Z. Ma, Solid State Commun. 150, 510 (2010).

${ }^{21}$ T. S. Nunner and F. von Oppen, Phys. Rev. B 84, 020405(R) (2011).

${ }^{22}$ A. Slachter, F. L. Bakker, and Bart Jan van Wees, Phys. Rev. B 84, 174408 (2011).

${ }^{23}$ B. Scharf, A. Matos-Abiague, I. Žutić, and J. Fabian, Phys. Rev. B 85, 085208 (2012).

${ }^{24}$ K. Uchida, S. Takahashi, K. Harii, J. Ieda, W. Koshibae, K. Ando, S. Maekawa, and E. Saitoh, Nat. Lett. 455, 778 (2008).

${ }^{25}$ K. Uchida, J. Xiao, H. Adachi, J. Ohe, S. Takahashi, J. Ieda, T. Ota, Y. Kajiwara, H. Umezawa, H. Kawai, G. E. W. Bauer, S. Maekawa, and E. Saitoh, Nat. Mater. 9, 894 (2010).

${ }^{26}$ C. M. Jaworski, J. Yang, S. Mack, D. D. Awschalom, J. P. Heremans, and R. C. Myers, Nat. Mater. 9, 898 (2010).

${ }^{27}$ C. M. Jaworski, J. Yang, S. Mack, D. D. Awschalom, R. C. Myers, and J. P. Heremans, Phys. Rev. Lett. 106, 186601 (2011).

${ }^{28}$ K. Uchida, T. Ota, H. Adachi, J. Xiao, T. Nonaka, Y. Kajiwara, G. E. W. Bauer, S. Maekawa, and E. Saitoh, J. Appl. Phys. 111, 103903 (2012).

${ }^{29}$ H. Adachi, K. Uchida, E. Saitoh, and S. Maekawa, arXiv:1209.6407.

${ }^{30}$ P. Schwab, R. Raimondi, and C. Gorini, Europhys. Lett. 90, 67004 (2010).
${ }^{31}$ M. I. Dyakonov, Phys. Rev. Lett. 99, 126601 (2007).

${ }^{32}$ N. Ashcroft and W. Mermin, Solid State Physics (Harcourt College Publishers, Orlando, FL, 1976).

${ }^{33}$ R. Raimondi, M. Leadbeater, P. Schwab, E. Caroti, and C. Castellani, Phys. Rev. B 64, 235110 (2001).

${ }^{34}$ P. Schwab and R. Raimondi, Eur. Phys. J. B 25, 483 (2002).

${ }^{35}$ R. Raimondi and P. Schwab, Phys. Rev. B 71, 033311 (2005).

${ }^{36}$ C. Gorini, P. Schwab, M. Dzierzawa, and R. Raimondi, Phys. Rev. B 78, 125327 (2008).

${ }^{37}$ R. Raimondi, P. Schwab, C. Gorini, and G. Vignale, Ann. Phys. (Berlin) 524, 153 (2012).

${ }^{38}$ R. Raimondi and P. Schwab, Europhys. Lett. 87, 37008 (2009).

${ }^{39}$ R. Raimondi and P. Schwab, Physica E 42, 952 (2010).

${ }^{40}$ S. Giglberger, L. E. Golub, V. V. Bel'kov, S. N. Danilov, D. Schuh, C. Gerl, F. Rohlfing, J. Stahl, W. Wegscheider, D. Weiss, W. Prettl, and S. D. Ganichev, Phys. Rev. B 75, 035327 (2007).

${ }^{41}$ J. L. Yu, Y. H. Chen, Y. Liu, C. Y. Jiang, H. Ma, and L. P. Zhu, Appl. Phys. Lett. 100, 152110 (2012).

${ }^{42}$ T. L. Hughes, Y. B. Bazaliy, and B. A. Bernevig, Phys. Rev. B 74, 193316 (2006).

${ }^{43}$ The parameter $\eta$ corresponds to what the authors of Ref. 42 call $\zeta$.

${ }^{44}$ T. Seki, Y. Hasegawa, S. Mitani, S. Takahashi, H. Inamura, S. Maekawa, J. Nitta, and K. Takanashi, Nat. Mater. 7, 125 (2008).

${ }^{45}$ H. Kontani, T. Tanaka, D. S. Hirashima, K. Yamada, and J. Inoue, Phys. Rev. Lett. 102, 016601 (2009).

${ }^{46}$ I. V. Tokatly, Phys. Rev. B 82, 161404(R) (2010).

${ }^{47}$ M. König, S. Wiedmann, C. Brüne, A. Roth, H. Buhmann, L. W. Molenkamp, X.-L. Qi, and S.-C. Zhang, Science 318, 766 (2007).

${ }^{48}$ Y. K. Kato, R. C. Myers, A. C. Gossard, and D. D. Awschalom, Science 306, 1910 (2004). 